\title{
Uncomplicated Pregnancy and Delivery after Previous Severe Postpartum Cerebral Angiopathy
}

\author{
Jan Rémi ${ }^{a}$ Thomas Pfefferkorn ${ }^{a} \quad$ Gunther Fesl $^{b}$ \\ Nina Rogenhofer ${ }^{c} \quad$ Andreas Straube $^{a} \quad$ Matthias Klein ${ }^{a}$ \\ Departments of ${ }^{a}$ Neurology, ${ }^{b}$ Neuroradiology and ${ }^{\mathrm{c}} \mathrm{Gynaecology}$, Klinikum \\ Grosshadern, University of Munich, Munich, Germany
}

\section{Key Words}

Postpartum cerebral angiopathy - Reversible cerebral vasoconstriction syndrome . Vasospasm $\cdot$ Stroke $\cdot$ Pregnancy

\begin{abstract}
Postpartum cerebral angiopathy (PCA) is a cerebral vasoconstriction syndrome developing shortly after delivery, without signs of preceding eclampsia. The risk for recurrence of PCA is unknown. Here, we report on a closely monitored, uneventful pregnancy of a woman with a previous severe episode of PCA. In summary, this case report demonstrates that PCA does not necessarily recur in following pregnancies, even after previous severe episodes.
\end{abstract}

\section{Introduction}

Postpartum cerebral angiopathy (PCA) is a cerebral vasoconstriction syndrome developing shortly after delivery, without signs of preceding eclampsia. The risk for recurrence of PCA is unknown. Here, we report on a closely monitored, uneventful pregnancy of a woman with a previous severe episode of PCA [1].

\section{Case Report}

A 32-year-old female patient was first treated at our university hospital when she developed a severe episode of PCA after delivery of her second child [1]. Briefly, she suffered from cerebral

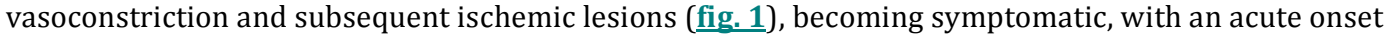
of very severe headache 4 days after delivery, left-sided hemianopia, and generalized tonic-clonic seizures. Signs of eclampsia were not present. Intra-arterial and subsequent intravenous nimodipine led to lasting improvement. Follow-up MRA showed a clear resolution of vasoconstriction 7 and 15 days after diagnosis. The patient was discharged without deficits, but was advised to have her status re-evaluated before a next pregnancy. 
Eight months after this first episode, the patient presented to our outpatient clinic being 6 weeks pregnant. She had no neurological complaints. An MRI showed only the previously documented lesions. Over the course of her pregnancy, no signs of vasoconstriction or epileptic activity were identified on repeated neurological examinations, EEGs, or MRIs and transcranial Doppler ultrasounds (TCD).

After 40 weeks of pregnancy, our patient had an uncomplicated vaginal delivery of a healthy boy. As during the second pregnancy, she had not received any medications having PCA as a possible side effect [2]. No neurological signs or symptoms occurred after delivery. Daily TCD from 3 days before to 10 days after delivery did not reveal signs of vasoconstriction. No new lesions (fig. 2 ) or signs of vasoconstriction were seen on MRI. At follow-up 6 months later, the patient was well without complaints.

\section{Discussion}

PCA is considered a rare, possibly underdiagnosed [2], but serious neurological complication in delivering women $[3,4]$. It is one of several reversible cerebral vasoconstriction syndromes (RCVS), characterized by reversible vasoconstriction of the cerebral arteries. RCVS are usually associated with an acute onset of thunderclap headache with or without neurologic deficits [4]. The pathophysiology of RCVS is thought to involve an acute change in cerebrovascular tone [2], which may be spontaneous or evoked by various exogenous or endogenous factors, with an uncertain pathophysiological mechanism [5-7]. It is not clear whether vasospasm, vasculitis or another mechanism or factor is the underlying reason for PCA [6, 8], but the pathophysiology may be similar to the disease mechanisms in eclampsia [9]. Since it is known that the risk of a repeated pre-eclampsia is more than twice as high as the risk in the general population, it is justified to consider that there is also an increased risk for repeated PCA in patients with PCA in a previous pregnancy [10].

For eclampsia, the rate of recurrence may be as high as 50\% [11], but for RCVS in general, and PCA in particular, these data are not available, especially since patients are typically advised to avoid the triggering factors [2]. In a literature review, we only found one report on the recurrence of PCA where a patient had severe intracranial hemorrhages after two consecutive pregnancies [12]. In comparison, our patient's course of her third pregnancy is encouraging, as it demonstrates no recurrence of PCA even after a previous severe episode of PCA.

Although conventional angiography is, by definition, the gold standard, non-invasive investigations such as MRI and CT of the brain and cerebral vessels have been largely used to support the diagnosis and follow-up. The results of the brain MRI are frequently normal in RCVS but can reveal evidence of infarction as well as parenchymal hemorrhages and small non-aneurysmal subarachnoid hemorrhages on the cortical surface. Furthermore, with the development of the MRI technique, the quality of the MRA rapidly becomes better.

PCA may recur; however, we do not know the rate of recurrence. Therefore, patients with pregnancies following an episode of PCA should be monitored closely. Due to the very limited number of cases and the lack of studies, it is unclear what surveillance of the patients would make sense. Of course, the basis for close monitoring are neurologic examinations on the days before and after delivery. Besides clinical monitoring, TCD seems an ideal tool to monitor patients for the development of cerebral 
vasoconstriction. TCD is non-invasive, has no side effects, is easy to perform, and is cheap. We chose to monitor our patient closely by TCD, checking for an increase of cerebral blood flow velocities monthly during the last 4 months of pregnancy and daily from 3 days before to 10 days after delivery. Furthermore, we recommend an MRA and MRI whenever clinical signs consistent with PCA develop (e.g. headaches, seizures, focal neurologic deficits). Since our patient had suffered from an epileptic seizure during PCA, we also performed an EEG the week before and after delivery to rule out epileptiform discharges.

For the treatment of severe episodes of PCA, calcium channel blockers are used, but only with little substantiating evidence $[1,13]$. Occurrence of subclinical signs of vasoconstriction on MRA or TCD may warrant consideration of oral nimodipine even before the onset of clinical signs.

Due to the lack of evidence, counseling patients with PCA with respect to following pregnancies is difficult compared to other gestational diseases [11], but our report is encouraging compared to a previous report on recurrence of PCA with most severe complications [12]. However, further studies are certainly needed to adequately understand the risk of a new pregnancy and to understand factors contributing to PCA. Until more comprehensive data on new pregnancies is available, close monitoring is recommended.

\section{Disclosure Statement}

No author has any conflict of interest to disclose. 


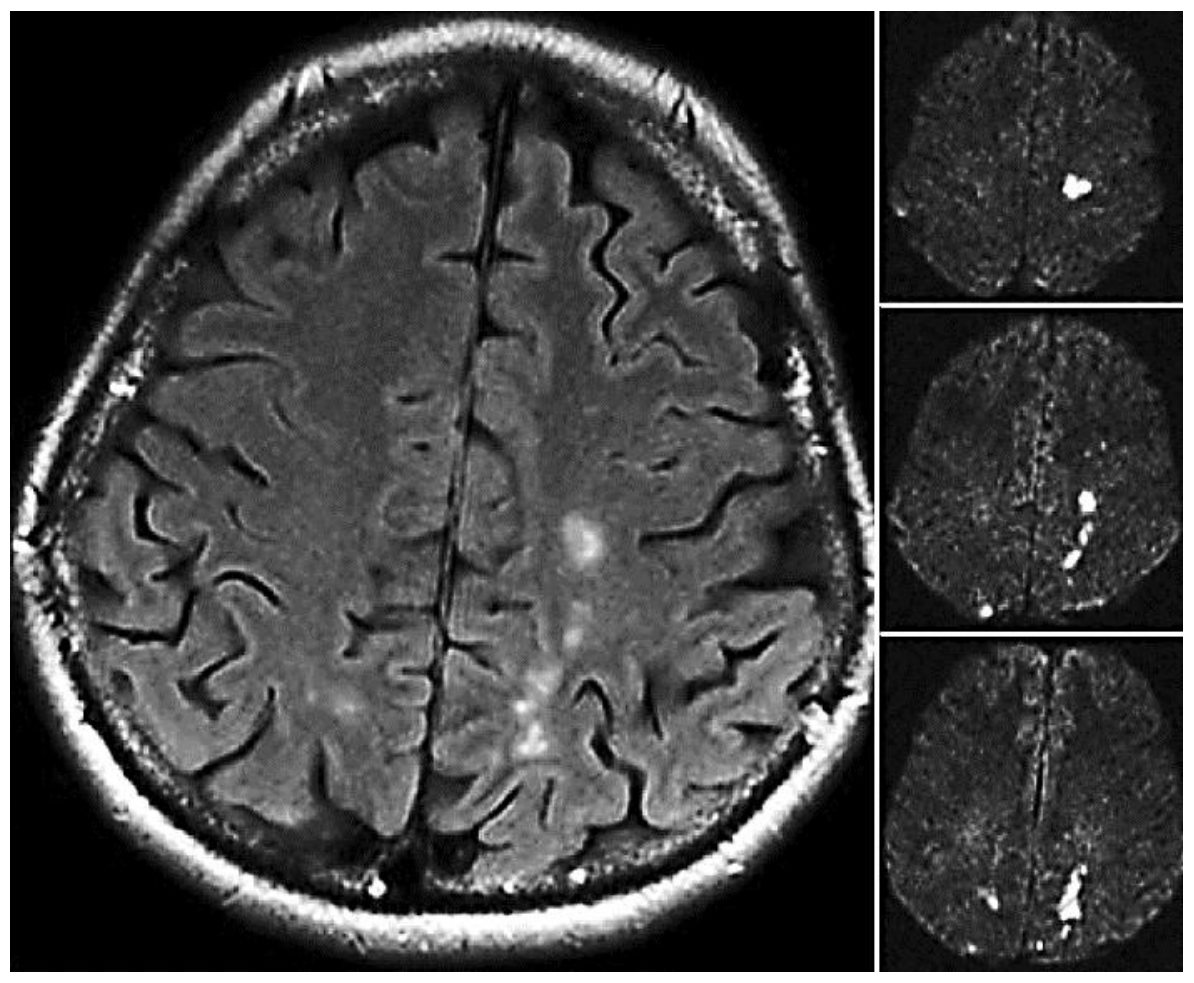

Fig. 1. MRI during the patient's previous episode of PCA [1]. FLAIR imaging (large image) and diffusion-weighted imaging (smaller images) show acute lesions in both parietal lobes and the left frontal lobe. 

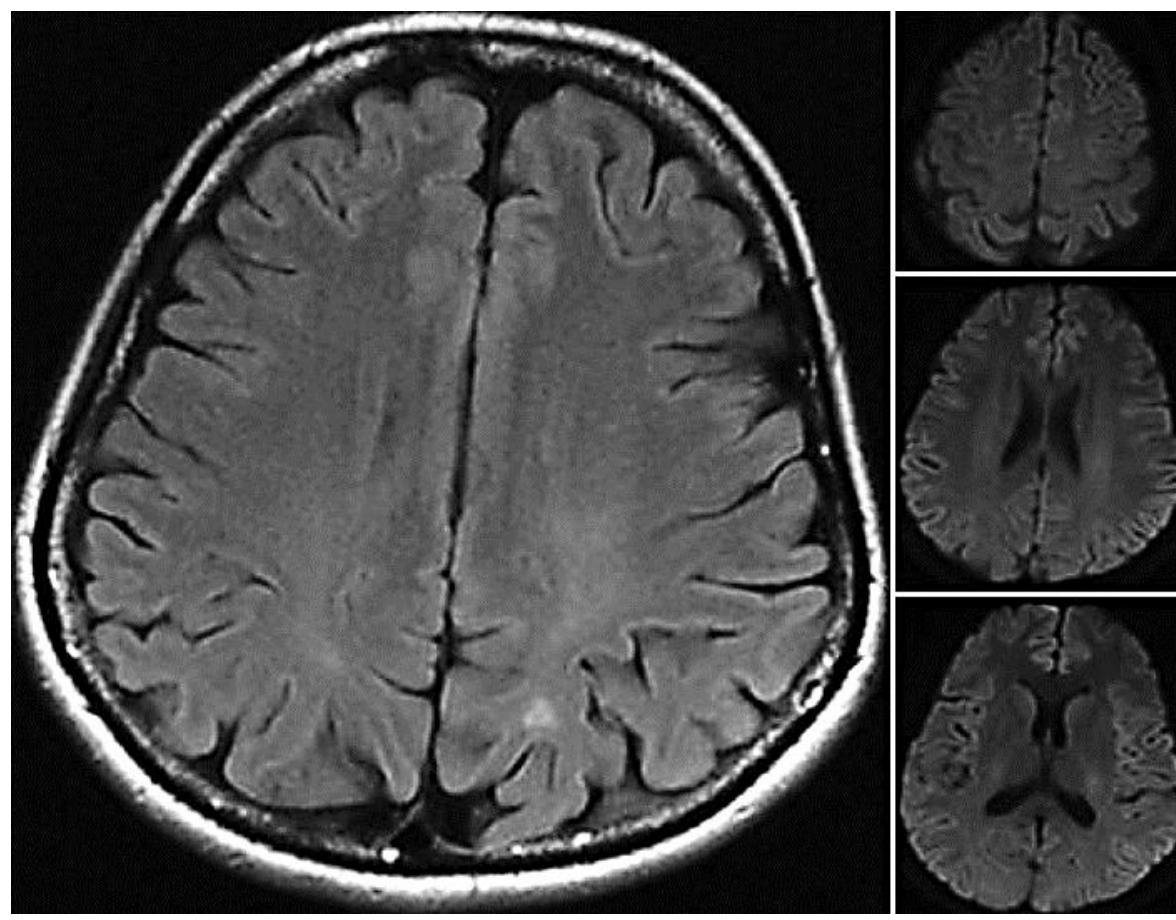

Fig. 2. MRI after the patient's last, uneventful pregnancy. Three days after delivery, FLAIR imaging (large image) shows the residual old lesions and diffusion-weighted imaging (smaller images) shows no new lesions.

\section{References}

1 Klein M, Fesl G, Pfister HW, Straube A, Bruckmann H, Hoffmann LA, Pfefferkorn T: Intra-arterial nimodipine in progressive postpartum cerebral angiopathy. Cephalalgia 2009;29:279-282.

$\checkmark 2$ Ducros A, Boukobza M, Porcher R, Sarov M, Valade D, Bousser MG: The clinical and radiological spectrum of reversible cerebral vasoconstriction syndrome. A prospective series of 67 patients. Brain 2007;130:3091-3101.

-3 Call GK, Fleming MC, Sealfon S, Levine H, Kistler JP, Fisher CM: Reversible cerebral segmental vasoconstriction. Stroke 1988;19:1159-1170.

4 Calabrese LH, Dodick DW, Schwedt TJ, Singhal AB: Narrative review: reversible cerebral vasoconstriction syndromes. Ann Intern Med 2007;146:34-44.

5 Tsukimori K, Ochi H, Yumoto Y, Iwasaki S, Hojo S, Noguchi T, Wake N: Reversible posterior encephalopathy syndrome followed by MR angiography-documented cerebral vasospasm in preeclampsia-eclampsia: report of 2 cases. Cerebrovasc Dis 2008;25:377-380.

$\checkmark 6$ Calado S, Vale-Santos J, Lima C, Viana-Baptista M: Postpartum cerebral angiopathy: vasospasm, vasculitis or both? Cerebrovasc Dis 2004;18:340-341. 
7 Oehm E, Hetzel A, Els T, Berlis A, Keck C, Will HG, Reinhard M: Cerebral hemodynamics and autoregulation in reversible posterior leukoencephalopathy syndrome caused by pre-/eclampsia. Cerebrovasc Dis 2006;22:204-208.

-8 Williams TL, Lukovits TG, Harris BT, Harker Rhodes C: A fatal case of postpartum cerebral angiopathy with literature review. Arch Gynecol Obstet 2007;275:67-77.

$>9$ Davie CA, O’Brien P: Stroke and pregnancy. J Neurol Neurosurg Psychiatry 2008;79:240-245.

-10 Tan CY, Ho JF, Chong YS, Loganath A, Chan YH, Ravichandran J, Lee CG, Chong SS: Paternal contribution of HLA-G*0106 significantly increases risk for pre-eclampsia in multigravid pregnancies. Mol Hum Reprod 2008;14:317-324.

11 Chames MC, Haddad B, Barton JR, Livingston JC, Sibai BM: Subsequent pregnancy outcome in women with a history of HELLP syndrome at $<$ or $=28$ weeks of gestation. Am J Obstet Gynecol 2003;188:15041507, discussion 1507-1508

12 Ursell MR, Marras CL, Farb R, Rowed DW, Black SE, Perry JR: Recurrent intracranial hemorrhage due to postpartum cerebral angiopathy: implications for management. Stroke 1998;29:1995-1998

-13 Calado S, Viana-Baptista M: Benign cerebral angiopathy; postpartum cerebral angiopathy: characteristics and treatment. Curr Treat Options Cardiovasc Med 2006;8:201-212. 